\title{
¿GOBIERNOS ABIERTOS EN ENTORNOS ANALÓGICOS? INICIATIVA DE GOBIERNO ABIERTO A NIVEL MUNICIPAL Y ESTATAL EN MÉXICO
}

\author{
Felipe J. Hevia \\ Centro de Investigaciones y Estudios Superiores en Antropología Social, \\ Golfo, México \\ fhevia@ciesas.edu.mx \\ Arturo Durán Padilla \\ El Colegio de Veracruz, México \\ jduran@colver.edu.mx
}

\begin{abstract}
RESUMEN
El gobierno abierto supone la existencia de una infraestructura tecnológica y la existencia de un gobierno y una sociedad con suficientes capacidades y habilidades digitales. Sin embargo, los datos disponibles para el sureste mexicano muestran déficits importantes en ambas dimensiones. Este artículo analiza si es posible desarrollar iniciativas de gobierno abierto en entornos que no presentan estas características. Tomando como estudio de caso la iniciativa local de Veracruz, en este artículo se presentan los resultados de la experiencia municipal de gobierno abierto y sus obligaciones de transparencia. Se concluye que es necesario considerar los marcos normativos y los compromisos en estos contextos, y promover ajustes e innovaciones para la apertura de los gobiernos en ámbitos con bajos recursos de conectividad.
\end{abstract}

Palabras clave: Gobierno municipal, Gobierno regional, Participación política, Reformas administrativas, México. 


\title{
OPEN GOVERNMENTS IN ANALOGICAL CONTEXTS? \\ OPEN GOVERNMENT INITIATIVE AT MUNICIPAL AND \\ STATE LEVEL IN MEXICO
}

\begin{abstract}
Open government assumes the existence of a technological infrastructure and the existence of a government and a society with sufficient digital capacities and abilities. However, the information available for south-east Mexico shows important deficits in both dimensions. This article analyzes whether it is possible to develop open government initiatives in contexts where these characteristics do not feature. Using the local initiative of Veracruz as a case study, this article presents the results of the municipal experience of open government and its transparency obligations. It is concluded that it is necessary to consider the regulatory frameworks and the commitments in these contexts, and to promote adjustments and innovations for the opening-up of governments in contexts of low connectivity resources.
\end{abstract}

Keywords: Municipal government, Regional government, Political participation, Administrative reforms, Mexico. 


\section{INTRODUCCIÓN Y PLANTEAMIENTO DEL PROBLEMA}

Desde que el presidente de Estados Unidos publicara su propuesta sobre gobierno abierto (Obama 2009) y se creara la Alianza por el Gobierno Abierto (OGP, por sus siglas en inglés) (OGP 2016), el interés académico y político sobre esta temática ha crecido de manera considerable. Existen cada vez más análisis centrados en el gobierno abierto a lo largo del mundo: Taiwan (Wang y Lo 2016), Estados Unidos (McDermott 2010), América Latina (Oszlak 2015), Brasil (Freitas y Dacorso 2014, Mesina 2013), y México (Valenzuela 2013, Sandoval-Almazán y Gil-Garcia 2016, Mesina 2013). De igual manera, se ha examinado al gobierno abierto desde perspectivas específicas, como son el fortalecimiento democrático (Ugalde 2015), la participación ciudadana (Sánchez 2015, Prieto-Martín y Ramírez-Alujas 2014), o sus efectos en las relaciones intragubernamentales (Valenzuela 2013).

Si bien, como múltiples autores señalan, el concepto de gobierno abierto no nació en el siglo XXI y su uso académico existe al menos desde la década de 1960 (Parks 1957), es sin duda que a partir de la primera década del presente siglo el concepto comenzó a despertar atención en la bibliografía especializada.

Una parte importante de la discusión al respecto se ha centrado en proponer una definición de lo que es, y no es, gobierno abierto (Wirtz y Birkmeyer 2015, Cruz-Rubio 2015, Calderón y Lorenzo 2010, Oszlak 2015, Sandoval-Almazan 2015, Sandoval-Almazan y Gil-Garcia 2016, Hofmann, Ramírez-Alujas, y Bojórquez 2012, Luna Pla, Bojórquez, y Hofmann 2015). De esta forma, el gobierno abierto puede definirse como una nueva idea o paradigma "que ubica al ciudadano en el centro de atención y de prioridad" (Cruz-Rubio 2015: 51), como "una plataforma tecnológica" (Sandoval-Almazan 2015: 214) o como "la noción de que las personas tienen el derecho de acceder a los documentos y procedimientos del gobierno [...] el derecho a escudrińar y participar en el gobierno" (Lathrop y Ruma 2010: IXI).

La mayoría de los conceptos que se presentan asocian la idea de gobierno abierto al desarrollo de las nuevas tecnologías de la información y comunicación (TIC). Así, por ejemplo, Calerdón y Lorenzo, en su libro pionero, afirman: "en su actual acepción [el GA] toma impulso y una importante cantidad de elementos del desarrollo del movimiento del software libre, el software de código abierto desarrollado por comunidades, hacker, puesto libremente a disposición de la comunidad y en permanente desarrollo y evolución" (Calderón y Lorenzo 2010: 13). 
Para Sandoval-Almazán "el gobierno abierto debe entenderse como una plataforma tecnológica institucional que convierta los datos gubernamentales en datos abiertos para permitir su uso, protección y colaboración por parte de los ciudadanos en los procesos de decisión pública, rendición de cuentas y mejoramiento de los servicios públicos" (Sandoval-Almazan 2015: 214). Para Claridad y Martínez el gobierno abierto se define por el uso de la tecnología de la información (Caridad y Martínez 2016: 50).

De esta forma, los ejes centrales del gobierno abierto -transparencia para promover rendición de cuentas, participación en la toma de decisiones y colaboración gobierno-sociedad, se relacionan directamente con las nuevas potencialidades que las TIC representan, incluyendo propuestas recientes que modifican esta triada tradicional, añadiendo dimensiones como "wikinomics" Estategia wiki-colaboración por medio de TIC-para los gobiernos; transparencia y $\mathrm{RdC}$, institucionalismo e "ingeniería social" (sociothecnical theory) y estado reticular (network state) (Sandoval-Almazan y Gil-Garcia 2016: 180). Así, esta tecnología, como los datos abiertos (Open data) podrían mejorar la gestión pública y rendición de cuentas (García 2014).

La inclusión de TIC en la gestión pública no es novedosa. Existe abundante bibliografía sobre el uso de estas tecnologías para la transparencia y el combate a la corrupción (Bertot, Jaeger, y Grimes 2010) o sobre gobierno electrónico (e-government) (Oszlak 2015). Sin embargo, para el caso de gobierno abierto su relación parece ser indisoluble. Desde su propio nombre, la idea de Gobierno Abierto como concepto (Oszlak 2015: 27-28), pertenece a esa generación de procesos abiertos como el Open Source Software movement (Lathrop y Ruma 2010: xix) y se asocia a innovaciones tecnológicas que llegan a confundirse con procesos políticos (Yu y Robinson 2012).

Así, muchas veces se emplea de manera indistinta a procesos como los movimientos de datos abiertos, gobierno abierto, y de web semántica para la gestión de la información, datos abiertos y vinculados (Quintanilla y Gil-Garcia 2016: 73). La inclusión del componente tecnológico, es tal que incluso Sandoval-Almazán afirma que:

Un gobierno abierto no podría ocurrir sin la tecnología. La mayoría de las investigaciones, casos de implementación, ejemplos prácticos estudiados en la revisión de literatura [...] mencionan el uso de la tecnología y en particular de las tecnologías de información y comunicación que se presentan a través de Internet como herramientas 
implícitas en la construcción de esta área de conocimiento (SandovalAlmazan 2015: 215)

Esta confusión entre innovaciones tecnológicas y procesos de gestión pública ha sido identificada como un problema o riesgo del desarrollo conceptual de gobierno abierto. De esta forma, por ejemplo, Calderón y Lorenzo, "cuando hablamos de Open Government estamos hablando fundamentalmente de valores, hablamos de repensar administraciones y gobiernos, sus procedimientos y sus dogmas" (Calderón y Lorenzo 2010: 14) a diferencia de las discusiones técnicas sobre Open data. Algo similar plantea Yu y Rodríguez, quienes, si bien valoran el nuevo vínculo de la tradición lejana de acceso a la información con nueva agenda de conectividad, advierten que el gobierno abierto no resuelve los problemas de participación ni gestión gubernamental, como tampoco mejora por sí mismo la transparencia (Yu y Robinson 2012).

El desfase que se aprecia en términos conceptuales entre innovación tecnológica y nueva propuesta de gestión pública que sugiere el GA, se expresa en las iniciativas emprendidas sobre la base de la Alianza por el Gobierno Abierto. Estudios sobre las iniciativas han encontrado, por ejemplo, como un problema "el sobredimensionamiento aún existente en el área tecnológica como espacio de coordinación, al igual que las temáticas destacadas de gobierno electrónico, poco centradas en la participación ciudadana” (Kaufman 2015: 69).

Bajo este estrecho vínculo, ¿Es posible el desarrollo de Gobiernos Abiertos en contextos donde la conectividad y acceso a las tecnologías de la comunicación no se han consolidado? ¿Puede pensarse en Gobiernos Abiertos en contextos analógicos, no digitales? Este es el problema de investigación que pretendemos revisar en este artículo: las dificultades $y$ potencialidades que esta estrategia enfrenta en contextos de baja conectividad.

A pesar de la extensa propaganda de proyectos y publicaciones, hoy no se encuentra suficiente información empírica que ayude a comprender las limitaciones y dificultades acerca del tema, pero también las potencialidades, que encierra este paradigma. Por ello, nuestro objetivo es mostrar cómo, en un estado del sureste de México, caracterizado por un contexto más analógico que digital, se han intentado desarrollar ejercicios de gobierno abierto y, con ello, iniciar la discusión en torno a sus resultados y sus implicaciones analíticas. 


\section{METODOLOGÍA}

Se determinó aplicar una metodología de estudio de caso (Yacuzzi 2005), por lo que se seleccionó a la Iniciativa de Gobierno Abierto de Veracruz. Este estado, debido a su peso demográfico, constituye la tercera entidad más poblada de México (INEGI 2011). Por sus condiciones socioeconómicas, es una de las regiones con mayor pobreza y desigualdad de acuerdo a las más recientes mediciones (CONEVAL 2015). Sus condiciones políticas están marcadas por administraciones estatales de baja calidad y capacidad (Hevia 2015), además prevalece una profunda crisis social, de derechos humanos y seguridad (Olvera, Zavaleta, y Andrade 2013). A pesar de las adversidades de este entorno, Veracruz fue una de las primeras entidades en promover un Plan de Acción Local de Gobierno Abierto (Hevia y Aguilera de Hombre 2015).

Dicho plan se diferenció de otras experiencias por construir "capítulos locales", correspondiendo cada uno a una serie de compromisos específicos. Uno de los cuales está a cargo del Órgano de Fiscalización Superior del Estado de Veracruz (ORFIS), el Colegio de Veracruz y Ciudadanos por Municipios Transparentes (CIMTRA).

El capítulo ORFIS-CIMTRA forma parte del Programa de Acción Local creado como un ejercicio de gobierno abierto. En este proyecto el conjunto de tareas se dirigió a verificar la información financiera publicada en los portales municipales del estado de Veracruz. El ejercicio se realizó teniendo como punto de partida la convocatoria a grupos ciudadanos y a la comunidad académica. Con ello, se pretendió impulsar la promoción de acciones de transparencia, dentro de las administraciones locales a partir de la definición de tres tareas colaborativas: 1) discutir y organizar una secuencia de procedimientos metódicos para verificar la información financiera en portales municipales; 2) articular la participación ciudadana con acciones de transparencia; y 3) proponer un modelo de un portal electrónico que permita, a las autoridades municipales, mayores recursos y oportunidades para el cumplimiento de sus obligaciones de transparencia.

Los antecedentes de este ejercicio se remontan a 2015. En ese año, el entonces Instituto Federal de Acceso a la Información y Protección de Datos (IFAI), acordó con la extinta Conferencia Mexicana para el Acceso a la Información Pública (COMAIP), impulsar ejercicios de gobierno abierto en las entidades federativas que de manera voluntaria accedieran participar.

Derivado de la invitación del Instituto Veracruzano de Acceso a la Información (IVAI), el Órgano de Fiscalización Superior del Estado de 
Veracruz accedió a colaborar en estos ejercicios locales, proponiéndose el compromiso de generar tareas para motivar el cumplimiento de las obligaciones de las autoridades municipales en materia de transparencia en sus páginas de Internet.

El ORFIS participó junto con otros estados de la República en una declaración conjunta para realizar trabajos a la promoción de una cultura ciudadana con capacidad para incidir en la atención de necesidades de interés público. Asimismo, la preparación del Capítulo ORFIS se complementó con la asistencia a tres talleres coordinados por el IFAI en marzo de 2015 (ORFIS 2016).

Este trabajo sirvió de base para el diseño del Plan de Acción Local. A partir de la declaratoria conjunta se instaló el Secretariado Técnico Local, conformando el ORFIS y CIMTRA el Capítulo 1 (Secretariado Técnico Local Veracruz 2015a). De este modo inició la etapa de difusión y reclutamiento buscando dar a conocer los ejercicios locales de gobierno abierto. Los resultados propiciaron la incorporación a este ejercicio de la comunidad del Colegio de Veracruz (Secretariado Técnico Local Veracruz 2015b).

En consecuencia, las actividades de promoción y reclutamiento permitieron discutir y reelaborar los compromisos originales para precisar como propósitos los siguientes: elaborar una metodología para la evaluación de información publicada en portales municipales; desarrollar una aplicación para los municipios que no cuenten con portal de Internet y coadyuvar en la instalación de CIMTRA-Capítulo Veracruz. Una vez seleccionado el caso, se procedió a buscar información en fuentes secundarias sobre los niveles de conectividad y habilidades digitales, con énfasis en los gobiernos municipales.

\section{Metodología DE VERIFICACióN}

El modelo metodológico del Capítulo ORFIS tuvo como pautas los estudios realizados desde el año 2009 por el equipo de trabajo de El Colegio de Veracruz en materia de análisis de portales de transparencia y de interactividad (Durán Padilla 2012, 2014a, 2014b, 2014c); la asistencia a talleres para la generación de indicadores coordinados por la Dirección Regional Oriente del Instituto Nacional de Estadística y Geografía (ORFIS 2016); y la realización de dos ejercicios de evaluación de información financiera en portales municipales elaborado por el Órgano de Fiscalización Superior del Estado de Veracruz (ORFIS 2016). 


\section{INSTRUMENTO DE VERIFICACIÓN}

Aunado a que la Ley 848 de transparencia del estado advierte la obligación de hacer pública la información en páginas web a municipios que cuentan con poblaciones de 70000 habitantes, puede encontrarse un grupo de códigos que exigen la difusión de información en la red omitiendo el criterio poblacional. A este marco jurídico se suman la Ley General de Contabilidad Gubernamental, la Ley de Coordinación Fiscal, la Ley de Presupuesto y Responsabilidad Hacendaria, así también la Ley General de Transparencia y Acceso a la Información Pública.

Por esta razón, en un primer momento, se diseñó una cédula para la observación que permitiera identificar particularidades de hospedaje y rasgos de interactividad de los portales municipales. Vale precisar que el hospedaje hace referencia al lugar específico donde se instala el portal institucional con una página de inicio, disponibilidad de servicios, información y contactos del municipio. Por su parte, la noción de interactividad procura aquí identificar las características que permiten a los usuarios solicitar información, gestionar servicios, así como los recursos disponibles para opinar, discutir y participar en las decisiones de la comunidad dentro del sitio electrónico. En una segunda etapa, a esta cédula de registro se sumaron las principales obligaciones normativas que deben cumplir las administraciones municipales.

En suma, la cédula de evaluación comprendió 114 rubros, los cuales buscaron revisar las características de los portales, así como el cumplimiento de las obligaciones de publicación derivadas de las siguientes legislaciones: Ley Número 848 de Transparencia y Acceso a la Información Pública para el Estado de Veracruz de Ignacio de la Llave (LTAIPEV); Ley General de Transparencia y Acceso a la Información Pública (LGTAIP) y Ley General de Contabilidad Gubernamental (LGCG).

El modelo aplicado comprende los siguientes componentes básicos. 
Tabla 1: Descripción instrumento de evaluación

\begin{tabular}{ccc} 
Módulo & Rubros por evaluar & Número de ítems \\
\hline 1 & $\begin{array}{c}\text { Características del portal } \\
\text { electrónico }\end{array}$ & 3 \\
Pautas de interactividad el \\
portal \\
Ley Número 848 de \\
Transparencia y Acceso \\
a la Información Pública \\
para el Estado de Veracruz \\
de Ignacio de la Llave \\
(LTAIPEV) la cual fue \\
abrogada por la Ley 875 \\
Ley General de \\
Transparencia y Acceso \\
a la Información Pública \\
(LGTAIP) \\
Ley General de \\
Contabilidad \\
Gubernamental \\
(LGCG), Título 5o De la \\
transparencia y difusión de \\
la información financiera.
\end{tabular}

Fuente: Elaboración propia.

El procedimiento de calificación fue el siguiente: Para cada uno de los ítems analizados se asignó la siguiente calificación: cero = no cumple; uno = cumple parcialmente $\mathrm{y}$; dos = cumple satisfactoriamente. Así, cada rubro tenía una calificación máxima posible si se cumplía satisfactoriamente cada ítem. La calificación final por ítem y por rubro se expresa en porcentajes sobre el valor máximo posible. Por ejemplo. El rubro "pautas de interactividad del portal" tiene cinco ítems, por lo tanto, su calificación máxima posible es 10 , que se expresa como $100 \%$. Si el municipio de Xalapa obtiene un $50 \%$, quiere decir que obtuvo cinco puntos sobre los 10 posibles.

Por último, se analizó información oficial sobre otros mecanismos y dispositivos de participación vinculados con la transparencia gubernamental.

\section{RESULTADOS}

Este apartado se compone de tres partes. En la primera se presentan los datos más sobresalientes respecto de la conectividad y capacidades digitales en los municipios de Veracruz. En la segunda se muestran los resultados de la medición a los portales de los ayuntamientos. La tercera se concentra en las implicaciones de los resultados, dando énfasis a la diferencia de tamaño 
entre los municipios analizados.

\section{Conectividad en Veracruz}

En Veracruz, los propósitos para mejorar la gestión administrativa de los ayuntamientos contrastan frente a los bajos niveles de capacitación con el que se desenvuelven las burocracias municipales. Al mismo tiempo, el incorporar equipamiento informático y dotar de tecnologías digitales a las comunidades, constituyen los retos por comunicar a una población poco mayor de 8.000.000 de habitantes residente en más de 22.000 localidades urbanas y rurales.

Sumado a tal contexto, los sistemas de comunicación de modo recurrente agrupan más recursos en las zonas centro y sur, en contraste con la región norte del estado, donde persisten problemas estructurales para proveer infraestructura carretera o dotar de redes de telefonía y acceso a Internet. Tal dispersión poblacional y distribución de recursos económicos condiciona un heterogéneo desarrollo (Durán Padilla 2014c: 31-32).

Al mismo tiempo, vale advertir que la infraestructura de recursos y la actividad de los servicios de transferencia de información electrónica están relacionados con sus componentes técnicos. Del mismo modo, la seguridad y el mantenimiento de estos sistemas dependen, en gran medida, de la incorporación de constantes ajustes e innovaciones.

Ello compromete dos tareas de los gobiernos locales. En un primer momento, implica erogaciones permanentes de gasto. En otro, se hace constante la actualización del personal encargado del manejo de información y de las operaciones contables del ayuntamiento. Estos constituyen aspectos relevantes para el presupuesto y la operación administrativa, sin embargo, no siempre resultan prioritarios en la organización de ayuntamientos tradicionales.

En México los usos tecnológicos presentan rasgos singulares. Destaca que el empleo de Internet para gestionar asuntos gubernamentales, comercializar o realizar operaciones bancarias en línea, representan todavía actividades marginales. En contraste, el principal consumo está dedicado a compartir información a través de los servicios de la red. De modo específico, los usos de mayor frecuencia reportados en México se concentran en buscar información escolar, comunicarse, obtener materiales audiovisuales, participar en redes sociales, consumir entretenimiento, auxiliar trabajos escolares, leer periódicos y libros, descargar programas o recursos digitales. 
Figura 1: Actividades en Internet, porcentajes (2015)

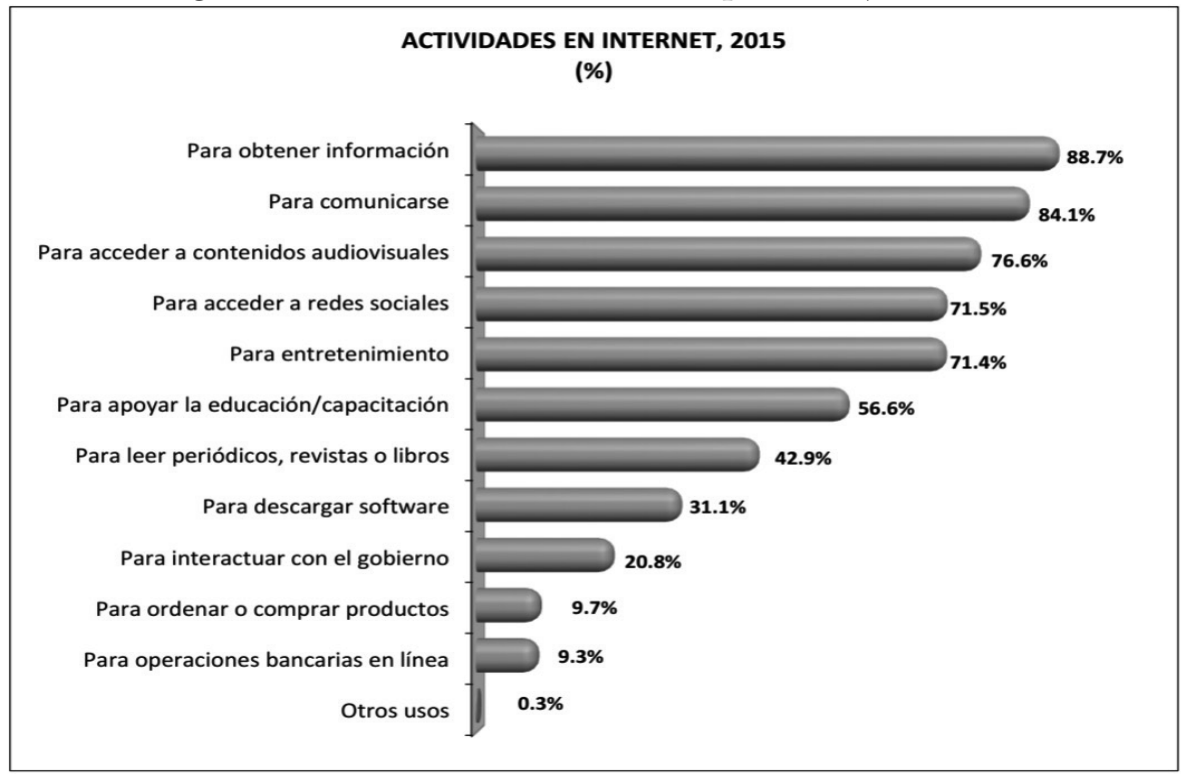

Nota: Las categorías no son excluyentes, por lo que la suma de las proporciones es superior al $100 \%$. Fuente: INEGI, ENDUTIH (2015).

Por otro lado, los problemas de conectividad se perciben como un fenómeno asociado a la indisponibilidad económica y a las limitaciones de la cobertura de la red de telecomunicaciones. La encuesta del Instituto Nacional de Geografía y Estadística (INEGI 2016) reporta que el 55,2\% de usuarios entrevistados consideran que "la falta de recursos" constituye el principal impedimento y que: "Entre las razones específicas (15,7\%) se identifica incluso la ausencia de infraestructura o proveedores del servicio en las localidades". Esto permite entender que la televisión constituye el recurso de mayor disponibilidad alcanzando el 93,5\%. En tanto, el uso de computadoras sin conexión comprende el 45\%, mientras que el acceso a Internet ocupa solo dos de cada cinco hogares.

En el caso del estado de Veracruz el contraste también es significativo. Siendo una de las entidades de mayor población, la conexión a servicios digitales presenta bajos rendimientos. Según los resultados de la Encuesta Nacional sobre Disponibilidad y Uso de la Tecnologías de la Información en los Hogares (ENDUTIH) 2015, el 57,4\% de la población de seis o más años en México se declaró usuaria de Internet, y que el 39,2\% de los hogares del país cuentan con conexión a Internet. Esta misma encuesta muestra diferencias regionales importantes. Así, mientras que el 64,5\% de los hogares de la Ciudad de México poseían una computadora, en el 
sureste mexicano los resultados fueron mucho menores: Chiapas 23,1\%, Oaxaca 24,6\% y Veracruz 31\% (INEGI 2016).

Respecto al número de usuarios de las tecnologías de información, el $51,3 \%$ de los mexicanos se consideró usuarios de computadora y el 57,4\% usuarios de Internet. Aquí también con diferencias importantes: mientras el 72\% de los habitantes de la Ciudad de México se consideraron usuarios de Internet, en Chiapas lo hizo solo el 33,7\%, en Oaxaca el $41,7 \%$ y en Veracruz el 49\% (INEGI 2016).

Como puede observarse, la variación al interior de cada entidad federativa es muy grande. La encuesta de INEGI no tiene representatividad subestatal, pero sí por ciudades específicas. Ahí, por ejemplo, se muestran variaciones menores. La ciudad de Tuxtla Gutiérrez (Chiapas) tiene un $63,1 \%$ de usuarios de Internet, la ciudad de Oaxaca (Oaxaca) tiene un $68,4 \%$ y la de Veracruz (Veracruz) un $70,7 \%$, no muy diferentes de la Zona Metropolitana del Valle de México, con un 69,8\%, o de Monterrey, con un $73,3 \%$ (INEGI 2016). Sin embargo, en zonas rurales, el porcentaje es cercano al $4 \%$ según reporta el Instituto del Derecho a las Telecomunicaciones (IDET 2015).

Las estrategias gubernamentales al respecto tampoco han logrado mejorar este panorama. Respecto a la estrategia de conectividad e-mexico, Durán concluye que:

Las conexiones de los centros rurales sin uso, evidenciaron el desconocimiento de las prioridades inmediatas y reales de la población [...] muchos de los telecentros instalados aún operan con limitaciones [...] El escaso aprovechamiento de e-mexico tiene varios orígenes [...] en la mayoría de la población existe poco conocimiento para conducirse por procedimientos electrónicos (Durán Padilla 2015: 97-98).

El simple acceso a Internet, como también se ha analizado, no garantiza su uso en términos de Gobierno Abierto. Profundizando datos de INEGI, Ugalde concluye que el "mayor número de usuarios de Internet no conduce necesariamente a que estos lo usen para interactuar con el gobierno" (Ugalde 2015: 86). Tal como se verá en el siguiente apartado, la baja conectividad se refleja también en la dificultad que tienen municipios medianos y pequeños para poder comunicarse con sus gobernados por medio de nuevas tecnologías, incluso las más básicas como la existencia de un portal en Internet. 


\section{PORTALES WEB MUNICIPALES Y TRANSPARENCIA}

Teniendo en consideración los resultados anteriores, no sorprende la baja cantidad y calidad de datos que los portales oficiales de los municipios en Veracruz entregan a los ciudadanos. De los 212 municipios en Veracruz, solo hay 24 municipios obligados a publicar información en portales web por contar más de 70.000 habitantes de acuerdo a la Ley de Transparencia, a saber: Veracruz, Xalapa, Coatzacoalcos, Córdoba, Poza Rica de Hidalgo, San Andrés Tuxtla, Tuxpan, Papantla, Minatitlán, Boca del Río, Cosoleacaque, Orizaba, Martínez de la Torre, Tierra Blanca, Álamo Temapache, Tantoyuca, Pánuco, Tihuatlán, Coatepec, Acayucan, Las Choapas, Emiliano Zapata, Medellín y Perote. Sin embargo, se encontraron 115 municipios con portales oficiales, correspondiendo al 54\% del total. De esta forma, en 2016 aún hay 97 municipios en Veracruz sin portales de Internet. De estos 97 municipios, se encuentran cuatro con más de 70.000 habitantes: Papantla, Álamo Temapache, Tihuatlán y Acayucan.

\section{Hospedaje}

De acuerdo al hospedaje de los portales vale destacar que la extensión .gob hace referencia a una entidad gubernamental, mientras que el sufijo .org identifica sitios de organizaciones sociales, y .mx corresponde a la región geográfica de sitios instalados en México. De este modo, pudo encontrarse que 106 corresponden a portales ubicados con extensión .gob; 4 a .com; 3 a .mx; 1 a .org; 1 a .or.mx, y 97 no presentan portal web.

El apartado dedicado a verificar las características generales de los portales dio cuenta de los rubros del calendario que fija la actualidad del día; el mapa de sitio; y el enlace activo que muestra las condiciones y reglas de seguridad. De los resultados obtenidos en este renglón, destaca que los mejores desempeños de hospedaje lo consiguieron solo cinco municipios, ninguno de los cuales es sujeto obligado por número de habitantes, que alcanzaron calificaciones superiores al 50\%. 
Tabla 2: Desempeño por hospedaje

\begin{tabular}{cc} 
Municipio & Portal electrónico \\
\hline Huayacocotla & $66,7 \%$ \\
Ozuluama de Mascareñas & $66,7 \%$ \\
Paso del Macho & $66,7 \%$ \\
Amatitlán & $50,0 \%$ \\
Ixhuatlán del Café & $50,0 \%$ \\
Zentla & $50,0 \%$ \\
& Fuente: Elaboración propia.
\end{tabular}

\section{Interactividad}

Respecto del criterio para observar el desempeño de interactividad, fueron considerados cinco características diferentes: marcadores de enlace para acceder a foros, chats o blogs; existencia de cajas de texto para escribir sugerencias; links para contestar encuestas; fechas o calendarios para la publicación de los resultados, así como referencias de actividad de redes sociales.

Al respecto, los municipios de Perote y Uxpanapa alcanzaron calificaciones notables superiores al 80\%, mientras que Banderilla, Paso del Macho, Xalapa, Martínez de la Torre y Soledad de Doblado lograron resultados suficientes. En consecuencia, 205 portales municipales en Veracruz no reúnen condiciones de una adecuada interactividad con los usuarios.

Tabla 3: Desempeño por interactividad

\begin{tabular}{cc} 
Municipio & Interactividad \\
\hline Perote* & $83,3 \%$ \\
Uxpanapa & $83,3 \%$ \\
Banderilla & $66,7 \%$ \\
Paso del Macho & $50,0 \%$ \\
Xalapa* & $50,0 \%$ \\
Martínez de la Torre* & $50,0 \%$ \\
Soledad de Doblado & $50,0 \%$
\end{tabular}

* Municipios con más de 70.000 habitantes. Fuente: Elaboración propia. 


\section{Marco estatal de transparencia}

Los criterios para estimar el funcionamiento de la transparencia en los portales municipales fueron considerados 72 rubros a partir de la Ley de transparencia estatal. Entre los aspectos atendidos, destacan los contenidos dentro del Artículo 8 en materia de normatividad, estructura administrativa, sueldos y salarios, planeación municipal, servicios, presupuestos, auditorías, enajenaciones, subsidios, licencias, inventarios de bienes, registros de solicitudes de información, condiciones laborales y de indicadores de desempeño de personal de policía.

De este modo, los resultados obtenidos presentan 13 portales con registros superiores al 50\%. Entre ellos, seis corresponden a municipios que cuentan con población superior a 70000 habitantes. Ello implica que más municipios medianos o pequeńos superan el rendimiento de los ayuntamientos obligados por la ley de transparencia estatal a publicar su información financiera de modo electrónico.

Tabla 4: Desempeño de transparencia estatal

\begin{tabular}{cc} 
Municipio & Transparencia \\
\hline Xalapa* $^{*}$ & $72,2 \%$ \\
Tuxpan* $^{*}$ Cerro Azul & $72,2 \%$ \\
San Andrés Tuxpla* & $67,4 \%$ \\
Perote* & $66,0 \%$ \\
Ixtaczoquitlán & $65,3 \%$ \\
Nanchital de Lázaro Cárdenas del Río & $63,9 \%$ \\
Minatitlán* & $60,4 \%$ \\
Tantoyuca* & $58,3 \%$ \\
Tepatlaxco & $56,9 \%$ \\
Ilamatlán & $54,2 \%$ \\
Citlaltépetl & $54,2 \%$ \\
Omealca & $52,8 \%$ \\
\end{tabular}

* Municipios con más de 70.000 habitantes. Fuente: Elaboración propia.

\section{Marco general de transparencia}

Este apartado revisa 13 asuntos derivados de los Artículos 8 y 70 de la Ley General de Transparencia y Acceso a la Información Pública. El contenido 
de asuntos atendidos se refiere a la solicitud de minutas del comité de transparencia municipal, relaciones de servidores públicos comisionados, indicadores del servicio de policía municipal, empleados con sanciones, montos de jubilados, gastos de comunicación social, encuestas a programas financiados con recursos públicos, actas del Comité de transparencia, contratos de servicios profesionales, resoluciones y laudos de juicios, relación de pensionados y declaraciones patrimoniales.

No obstante que fueron 13 asuntos por verificar de la Ley General de Transparencia y Acceso a la Información Pública, únicamente 15 municipios reportaron información sin rebasar el 50\% de cumplimiento esperado. Del grupo de los 15 portales, el caso de mayor desempeño alcanzó el 46\%, en tanto que los menores fueron registrados con solo 3,8\%. En suma, únicamente 4 municipios con más de 70.000 habitantes presentaron información solicitada por la Ley General de Transparencia y Acceso a la Información Pública, en contraste a los 11 portales restantes.

Tabla 5: Desempeño por ley general de transparencia

\begin{tabular}{cc} 
Municipio & LGTAIP (13) \\
\hline Perote* $^{*}$ & $46,2 \%$ \\
Minatitlán* $^{*}$ Tuxpan* $^{*}$ & $30,8 \%$ \\
Ilamatlán & $23,1 \%$ \\
Tepatlaxco & $19,2 \%$ \\
Veracruz & $15,4 \%$ \\
Huatusco & $15,4 \%$ \\
Tehuipango & $15,4 \%$ \\
Paso del Macho & $15,4 \%$ \\
Cerro Azul & $15,4 \%$ \\
Ixtaczoquitlán & $7,7 \%$ \\
Citlaltépetl & $7,7 \%$ \\
Omealca & $7,7 \%$ \\
Las Minas & $7,7 \%$ \\
Naranjal & $7,7 \%$ \\
${ }^{*}$ Municipios con más de 70.000 habitantes. & Fuente: Elaboración propia.
\end{tabular}




\section{Contabilidad gubernamental}

Ley General de Contabilidad Gubernamental se estableció fijando las pautas para la organización de información financiera de las entidades públicas a fin de armonizar la fiscalización del gasto e ingreso públicos. El órgano encargado para coordinar el proceso es el Consejo Nacional de Armonización Contable (CONAC).

Las modificaciones en materia de transparencia de recursos y de procesos impactaron sobre la obligación de publicación de normas locales. Por este motivo, el ejercicio se amplió considerando 21 rubros para conocer los estados financieros, los ejercicios de egresos, las acciones de armonización contable, así como diversas tareas para la difusión de sus procesos y resultados a las autoridades hacendarias y a la ciudadanía.

En suma, de los 115 municipios con portal, solo seis municipios que presentaron información financiera relativa a los criterios del marco de la contabilidad general. De estos, solo tres de ellos generaron un desempeño superior a la media general. En contrapartida, los otros tres sitios tuvieron un limitado rendimiento alcanzando calificaciones del 38\% al 9,5\%.

Tabla 6: Desempeño por normatividad de contabilidad gubernamental

\begin{tabular}{cc} 
Municipio & $\begin{array}{c}\text { Gestión financiera } \\
\text { Artículo 48 LGCG }\end{array}$ \\
\hline Juan Rodríguez Clara & $76,2 \%$ \\
Xalapa* $^{*}$ & $69,0 \%$ \\
Nogales $^{*}$ Córdoba & $57,1 \%$ \\
Naolinco $^{*}$ & $38,1 \%$ \\
Veracruz $^{*}$ & $33,3 \%$ \\
& $9,5 \%$ \\
& Municipios con más de 70.000 habitantes. \\
& Fuente: Elaboración propia.
\end{tabular}

Resultados agregados

La siguiente tabla resume los resultados agregados de los indicadores que se analizaron. 
Tabla 7: Resultados generales portales municipios Veracruz

\begin{tabular}{|c|c|c|}
\hline Posición & Municipio & Calificación general \\
\hline 1 & Xalapa* & $61,3 \%$ \\
\hline 2 & Perote* & $51,3 \%$ \\
\hline 3 & Tuxpan* & $49,6 \%$ \\
\hline 4 & Cerro Azul & $45,2 \%$ \\
\hline 5 & San Andrés Tuxtla* & $43,0 \%$ \\
\hline 6 & Minatitlán* & $42,6 \%$ \\
\hline 7 & Ixtaczoquitlán & $42,6 \%$ \\
\hline 8 & $\begin{array}{l}\text { Nanchital de Lázaro } \\
\text { Cárdenas del Río }\end{array}$ & $38,7 \%$ \\
\hline 9 & Tantoyuca* & $37,0 \%$ \\
\hline 10 & Ilamatlán & $36,5 \%$ \\
\hline \multirow[t]{2}{*}{11} & Tepatlaxco & $36,5 \%$ \\
\hline & ${ }^{*}$ Municipios c & $\begin{array}{l}\text { de } 70.000 \text { habita } \\
\text { te: Elaboración pro }\end{array}$ \\
\hline
\end{tabular}

De esta forma, los resultados muestran que el mejor desempeño de los portales no está relacionado con el tamaño de los municipios. Cada uno de los rubros analizados, y los resultados en general, muestran amplia variabilidad en el desempeño de municipios "grandes" (de 70.000 habitantes) y "pequeños" (de menos de 70.000 habitantes). Solo considerando los 11 mejores desempeńos, vemos que, si bien las medias de los municipios grandes son más altas $(46,6 \mathrm{DE}=8,33)$ que los municipios menores $(41,1 \mathrm{DE}=6,21)$, haciendo una prueba de T-Student estas diferencias resultaron estadísticamente no significativas. Esta variabilidad es importante no solo porque los municipios grandes tienen la obligación legal de dar la información analizada, sino también porque son estos mismos municipios los que cuentan con mayores presupuestos.

\section{ANÁLISIS DE LAS IMPLICACIONES DE LOS RESULTADOS OBTENIDOS}

Al principio de este trabajo se señaló que las distintas perspectivas dedicadas al estudio de las políticas de gobierno abierto presuponen la convergencia de tres condiciones básicas: la participación abierta de la ciudadanía; el 
manejo transparente de los patrimonios y de los servicios públicos; así como, el empleo de los recursos tecnológicos de la comunicación. De alguna u otra manera, para distintos autores, esto implica la exigencia y coincidencia de estos tres componentes o, en otros casos, la preponderancia de algunos de ellos.

Frente a ello, el planteamiento original de esta investigación ha cuestionado acerca de las posibilidades de desarrollo de los gobiernos abiertos en ambientes donde persisten condiciones precarias al acceso de las tecnologías de la comunicación. Por nuestra parte, los resultados de esta evaluación destacan la importancia de los canales tecnológicos como facilitadores para la difusión de información gubernamental, sin embargo, estos no constituyen elementos condicionantes para el cumplimiento de las obligaciones normativas que regulan la visibilidad de los recursos públicos.

Distante de las expectativas consideradas por los diversos enfoques de gobierno abierto, el balance de este ejercicio anticipa distintas tendencias poco alentadoras dentro de los grandes rubros de participación ciudadana, acceso al manejo transparente de información y usos de recursos tecnológicos de comunicación.

Respecto del primer punto, participación ciudadana, de 212 municipios evaluados, 108 de ellos no muestran mecanismos ni procedimientos que permitan la comunicación directa y efectiva entre los administradores del sitio con sus usuarios. Mucho menos se posibilita la formación de foros donde puedan generarse discusiones colectivas de los ciudadanos. Destacan algunos esfuerzos municipales por mantener información actualizada e incorporar procesos favorables a la rendición de cuentas como talleres colectivos y algunas estrategias de difusión, sin embargo, solo dos portales obtuvieron calificaciones superiores al $80 \%$; y 5 únicamente lograron resultados suficientes.

Aunque el estudio trató de identificar condiciones propicias para las solicitudes de información y la gestión de servicios, únicamente se identificaron foros eventuales y encuestas sin periodicidad. Al mismo tiempo, destaca que en ninguno de ellos pudieron encontrarse mecanismos ni procedimientos que permitan a sus usuarios participar en la toma de decisiones.

Respecto del segundo punto, acceso transparente de información y servicios públicos, aun contando con amplios recursos los gobiernos no han establecido, por ellos mismos, políticas de gobierno abierto. La instalación de estos sistemas se ha generado más por normas generales y 
exigencias exógenas, que de iniciativas originadas para atender condiciones y demandas de las comunidades locales. Por ello, a pesar de que el conjunto de normas regulatorias establece obligatoria la difusión financiera de los 212 municipios de Veracruz, solo la mitad dispone de portales electrónicos. Persisten oportunidades legales y apoyos estatales, pero tales respaldos no se ven reflejados en las acciones municipales.

Las evidencias indican un bajo cumplimiento aún entre municipios que cuentan con recursos y poblaciones mayoritarias. En este ejercicio solo 6 de los municipios presentaron información financiera relativa al marco de la contabilidad gubernamental. De ese total, 3 mostraron un desempeño superior a la media general y los restantes tuvieron un rendimiento limitado.

Destaca la recurrencia de prácticas poco institucionales. Algunos administradores de portales, para simular el cumplimiento de obligaciones de transparencia, adoptan pautas contrarias al desempeńo institucional colocando enlaces que remiten a sitios de organismos reguladores de la transparencia pública. Como resultado, la información disponible no cuenta con calidad suficiente. La mayoría de portales instalados carece de continuidad o periodicidad de publicación y su información no alcanza a cumplir satisfactoriamente los requisitos que exigen las diferentes normas de transparencia.

Por último, respecto del empleo de los recursos tecnológicos, se concluye lo siguiente: no obstante que los 212 ayuntamientos de Veracruz están en condiciones para instalar y operar portales de transparencia y cumplir las obligaciones de los diversos procesos regulatorios, el $50 \%$ de sitios están inscritos bajo la extensión .gob.mx, mientras el $4 \%$ se encuentra alojado entre diversos servidores comerciales o particulares, lo cual representa riesgos para la continuidad de las políticas locales de información, al quedar los sitios bajo el control de quienes promovieron su registro. Aunado a que la contratación de servicios tecnológicos, es posible que el gobierno estatal brinde apoyos de hospedaje gratuito, a la vez proporciona asistencia técnica para administrar los portales y capacitación para el ordenamiento de información financiera sujeta a publicarse.

Si en su caso las administraciones municipales presentan dificultades técnicas o carencias de recursos económicos, mucho de la precariedad tecnológica se concentra en la población de usuarios. Las situaciones de pobreza unidas a la dispersión geográfica marcan prioridades distintas a las necesidades de transparencia.

En suma, el cumplimiento de las normas de transparencia y la apertura de datos no mantienen necesaria correspondencia con la ubicación 
geográfica, el número de pobladores o los presupuestos asignados. El argumento común que justifica el incumplimiento parte de cuestionar para qué habilitar un complejo sistema que no será consultado. Al mismo tiempo, tal situación retroalimenta un sistema carente de recursos técnicos, donde prevalece la falta de disposición administrativa para transparentar la información pública.

Puede decirse, entonces, que hoy los proyectos de datos abiertos y de participación ciudadana en ámbitos locales, enfrentan problemas de baja calidad de la información, poca disposición ciudadana y una precaria colaboración institucional. Por ello, los distintos enfoques deberían incluir entre sus agendas de discusión los aspectos técnicos, así como los análisis de los contextos donde se desarrollan los programas de gobierno abierto.

\section{DISCUSIÓN Y CONCLUSIONES}

Los resultados generales alcanzados en este ejercicio sugieren la necesidad de pensar cómo crear una agenda de estrategias alternativas que permita a las iniciativas ciudadanas en contextos analógicos transitar o escalar hacia etapas que posibiliten aprovechar las potencialidades de la apertura gubernamental. Nuestra opinión considera la pertinencia de reflexionar los contenidos de esta agenda en torno a tres dimensiones que deberían discutirse de manera interrelacionada. La primera dimensión la constituye una relación técnica compuesta de las condiciones de recursos y cobertura de redes para la transmisión de información pública. La segunda, se vincula a una estructura jurídico administrativa de la vida local ante la incorporación de nuevas racionalidades normativas. Mientras la tercera dimensión tiene que ver con la esfera de la política tradicional.

Para discutir la dimensión técnica cabe anticipar que, en la sustitución de pautas de usabilidad analógica, existen profundas dificultades. La promesa del empleo de mecanismos técnicos está dirigida a garantizar mayor eficiencia de los recursos empleados. Sin embrago, frente a los propósitos por obtener resultados eficientes bajo la operación de plataformas de servicios automatizados, el paradigma fundado en la dependencia tecnológica implica la instalación de un sistema de mecanismos complejos.

El problema de este modelo de administración, por un lado, se basa en el sometimiento a una constante renovación tecnológica, lo cual conlleva a una permanente e interminable sustitución de insumos y procedimientos técnicos. Por otro lado, las innovaciones electrónicas no operan en relación a necesidades locales sino, cada vez más, a competencias de mercados globalizados. Debido a ello, la constante renovación de infraestructura genera la coexistencia de distintas generaciones de equipos perecederos y la 
multiplicidad de códigos y protocolos de conexión sustituibles.

La misma lógica de competencia del mercado y del consumo electrónico reúne diversas condiciones propicias a la incompatibilidad de los sistemas de comunicación y, contradictoriamente con ello, contribuye a retardar el desarrollo de una transparencia de mayor calidad. Así, la comunicación resulta compleja al yuxtaponerse distintas generaciones tecnológicas. Los equipos precarios no alcanzan a enlazarse debido a su insuficiente capacidad de rendimiento frente a las altas demandas de recursos de plataformas por las que se distribuye información. Tal incompatibilidad y limitaciones hacen que muchos usuarios se cuestionen el uso técnico cuando con lentitud, pero con relativa satisfacción, los servicios directamente eran brindados en las ventanillas.

El contexto revela una condición inevitable. En una inadecuada incorporación tecnológica, los usuarios cuentan con mayor cantidad de información, pero no necesariamente tienen a su alcance instrumentos suficientes para interpretarla. En tales circunstancias, los usuarios disponen de un mayor número de recursos para realizar trámites, pero no siempre aprovechan con integridad los servicios de información. Por su parte, los administradores pueden agrupar más medios automáticos, aunque no necesariamente se desempeñan mejor. Pensado de otro modo, hoy mucho de la acumulación de chatarra electrónica dibuja metafóricamente la imagen futura de los proyectos de modernización administrativa.

Insistimos, no se quiere afirmar aquí que lo técnico impida elevar la calidad del servicio público. Solo pretende advertirse que lo técnico no debe constituir la única vía para construir un gobierno abierto, participativo, transparente y cooperativo, dadas las preocupantes evidencias que reclaman revisar, de origen, el planteamiento de los programas para la reorganización de la gestión pública. En consecuencia, ni lo analógico ni la precariedad de recursos deberían constituir inconvenientes para limitar el cumplimiento de los principios de disponibilidad, prontitud y veracidad de la información que deben presentar los gobiernos a sus ciudadanos. En la relación entre la vida local y el consumo tecnológico, lo digital continúa siendo una anhelada promesa y lo analógico una fracturada realidad.

Una segunda dimensión en esta agenda se presenta asociada a la esfera administrativa y al marco normativo de la vida local. Vale anotar al respecto que durante las últimas tres décadas los sistemas tradicionales de administración pública se han enfrentado a grandes dificultades motivadas, en muchos de los casos, por la creciente demanda de servicios frente a un preocupante y continuo deterioro de los tradicionales procedimientos. 
Ante esta particular condición, la demanda de sistemas más productivos y la aparición de nuevos patrones de transparencia y participación ciudadana también se han incrementado.

Por ello, la operación de una política de gobierno abierto se convierte en una oportunidad para crear plataformas administrativas integradas. Ello se traduce en planes organizados, interfaces de consulta y actualización, racionalización de insumos, renovación constante de recursos, empleados capacitados, vínculos con los usuarios y capacidad para atender inquietudes de los solicitantes. Al mismo tiempo, presupone la actualización y adaptación a estándares nacionales y la promoción de usos adecuados de información. La agenda de asuntos es amplia. De un lado, en el plano político administrativo aún queda por revisar la indisposición política de las autoridades y representantes municipales, las formaciones de los cuadros administrativos del ayuntamiento. En el otro, se requiere indagar la manera en que las carencias de recursos técnicos, así como la falta de voluntad política impiden discutir y participar de los asuntos comunitarios.

En contraste, vale recordar al respecto que los municipios operan, en la mayoría de las ocasiones, con resistencias sujetas a prácticas arraigadas. De ese modo, la prestación de servicios y la relación con los ciudadanos, dependen en gran medida de estructuras basadas en los usos y costumbres de la gestión tradicional. Por ello, no resultan ajenas las inercias autoritarias, las disputas entre grupos o prácticas de clientelismos entre las unidades administrativas. Este ambiente favorece la fragmentación de funciones y la descoordinación de los trabajos del ayuntamiento. Aunado a ello, prevalece cada vez más una tendencia que impone la sobrerregulación normativa de códigos complejos y exógenos propiciando la burocratización de los servicios y la reducción de la capacidad de atención local.

Con todo el establecimiento de los programas de gobierno abierto depende en gran medida de la estructura que los establece y administra. En la mayoría de los casos, al ser impulsados como proyectos sobre formas institucionales sedimentadas, se corren el riesgo de que estas iniciativas terminen adaptándose a los intereses y voluntades de los sistemas que los opera. Por otro lado, y es oportuno reconocerlo, comienza a generalizarse una clara tendencia inapropiada en la coordinación de los ejercicios de evaluación municipal. Esta se expresa en una insistente aplicación de pautas de seguimiento y evaluaciones que hacen, del propósito de cumplimiento, una extenuante tarea que ahoga el funcionamiento y la eficiencia de los servicios locales ya de por si precarios.

Contrario a la formulación de una administración abierta, de no advertir de los riesgos nuevamente se estaría promoviendo la formación de una 
burocracia con tendencias centralizadas. Aún más, el modelo de gobierno abierto no ha probado su eficiencia y productividad. Hoy se celebran las iniciativas que anuncian la renovación de las estructuras administrativas y la reorganización del servicio público y se abona la esperanza de un mejor futuro para este modelo de gestión. Sin embargo, el optimismo está omitiendo que una estructura de este tipo, sin equilibrios ni contrapesos de vigilancia, corre el riesgo de transformarse en un sistema contrario. En esta lógica, no resultará casual que algunos recursos tecnológicos de la información sean utilizados por gobiernos demagógicos, que encuentren nuevas alternativas para abusar del gasto público o beneficiarse de influencias políticas. Desde distintos puntos de vista, algunas evidencias llaman atención para mantener mayores reservas y actitudes más prudentes. El riesgo radica en el afianzamiento de una administración orientada más por intereses y a favor de las opacidades.

La tercera dimensión de esta agenda de transición de lo analógico a lo digital, tiene que ver con las características que las relaciones sociedadestado en general adoptan en estos contextos. Existen investigaciones que apuntan hacia la diversidad de patrones o dinámicas de relación sociedadEstado en Veracruz y a la construcción de capacidades estatales de respuesta diferenciada (Hevia 2015). En el ámbito municipal, a pesar de su enorme variación y capacidades institucionales, las dinámicas hegemónicas de relación tienen que ver con las relaciones personalistas que se establecen entre los munícipes y la población (Grindle 2007). Estas relaciones personalistas, no están reñidas en principio con una mayor interactividad, al contrario, las fomentan. La posibilidad de interacción directa que ofrecen redes sociales como Facebook y Twitter permite esta atención cercana y esta interacción directa sin intermediación política.

Pero, como se advierte para el caso educativo (Hevia 2014), este tipo de relaciones incrementa la asimetría de poder entre gobernantes y gobernados puesto que aumentan los niveles de discrecionalidad e incertidumbre, tal como lo describiera Crozier (1969) hace casi cincuenta años. Así, la transición hacia un gobierno abierto implica también modificar estos patrones de relación y fomentar más bien acciones que generen certidumbre entre los gobernados, que permitan el establecimiento de rutinas, que amplíen sus posibilidades de voz y acceso a la toma de decisiones, y que incrementen la colaboración. Nada de esto depende exclusivamente de la conectividad, y requiere, por tanto, de modificaciones institucionales, en su amplio sentido (Ostrom 2015) que hagan del gobierno abierto menos una nueva utopía y más una serie de compromisos factibles para tener mejores servicios públicos y gobiernos más democráticos. 
El balance general de este ejercicio sugiere que el debate en torno al destino de los gobiernos abiertos se ha quedado estacionado en las definiciones de los conceptos, en la búsqueda de atributos y ventajas de los programas, así como en las convocatorias y en los alcances de la participación ciudadana. Finalmente, por nuestra parte consideramos que el círculo virtual de un proyecto de gobierno abierto compromete por lo menos tres momentos de un mismo proceso.

Una primera fase resulta de carácter propositivo; otra, de acción y construcción del proyecto y; una tercera, de realización y consolidación del programa. En específico, la primera comprende el desarrollo de iniciativas para poner en práctica acciones ciudadanas de apertura gubernamental. Un segundo momento aparece relacionado a la disposición de las instituciones para aceptar la interlocución con la ciudadanía y, en conjunto, impulsar la adopción y ejecución de acciones colaborativas. La tercera y definitiva etapa implica el establecimiento de estos procedimientos dentro de la operación cotidiana de las estructuras públicas bajo una permanente vigilancia por parte de instancias y especialistas ciudadanos.

No obstante, las potencialidades que ofrece este círculo están pendientes de alcanzarse. Por ello, la principal consideración de este ejercicio llama la atención para continuar discutiendo acerca de los contenidos de los programas de gobierno abierto, revalorar sus compromisos y consecuencias en donde la expectativa de conectividad técnica no ha logrado realizarse. Ello implica, como habíamos anticipado, promover ajustes e innovaciones para que la apertura de los gobiernos en ámbitos con bajos recursos de conectividad resulte exitosa. 


\section{REFERENCIAS}

Bertot, J., Jaeger, P. y Grimes, J. (2010). Using ICTs to Create a Culture of Transparency: E-Government and Social Media as Openness and AntiCorruption Tools for Societies. Government Information Quarterly, 27 (3), 264-271.

Calderón, C. y Sebastián Lorenzo, S. (2010). Introducción. En Calderón, C. y Sebastián Lorenzo, S. (Ed.), Open Government: Gobierno Abierto. Jaén: Algón Editores.

Caridad, M. y Martínez, S. (2016). Gobierno y Parlamento Abierto: La Participación Ciudadana en el Tratamiento y Visualización de la Información Pública. Revista Interamericana de Bibliotecología, 39 (1), 47-56.

Consejo Nacional de Evaluación de la Política de Desarrollo Social (CONEVAL). (2015). Medición de la Pobreza en México y en las Entidades Federativas. 2014. México: CONEVAL.

Crozier, M. (1969). El Fenómeno Burocrático 2. Ensayo sobre las Tendencias Burocráticas de los Sistemas de Organización Modernos y sus Relaciones con el Sistema Social y Cultural. Buenos Aires: Amorrortu.

Cruz-Rubio, C. (2015). “¿Qué Es (y Que No Es) Gobierno Abierto? Una Discusión Conceptual. EUNOMÍA. Revista en Cultura de la Legalidad, $8,37-53$.

Durán Padilla, A. (2012). Gestión Electrónica y Transparencia en gobiernos locales. La Experiencia en Municipios de Veracruz 2011. En De la Peńa, G. y Gervasi, F (Eds.), Memorias XIV encuentro nacional de la AMIC. Coahuila: UAC, AMIC.

(2014a). Dificultades Teóricas en Gestión Electrónica en Gobiernos Locales. En Memorias del 6to Congreso Internacional de Sociología. La Paz: Universidad Autónoma de Baja California.

. (2014b). Metodologías en la Comunicación y Gestión Electrónica en Gobiernos Locales. En Memorias del Congreso Democracia, Comunicación Y Movimientos Sociales. México: UAEM.

(2014c). Rendición de Cuentas y Gestión Electrónica en Gobiernos Locales. En La Construcción del Futuro: Los Retos de 
las Ciencias Sociales en México. Memorias del $4^{\circ}$ Congreso Nacional de Ciencias Sociales. México: Congreso Nacional de Ciencias Sociales.

IIJ-UV. . (2015). Estado y Regulación de la Red en México. Xalapa:

Freitas, R., y Rocha Dacorso, A. (2014). Inovação Aberta na gestáo Pública: Análise do Plano de Ação Brasileiro para a Open Government Partnership. Revista de Administraçâo Pública 48 (4), 869-888.

García, J. (2014). Gobierno Abierto, Transparencia, Participación y Colaboración en las Administraciones Públicas. Innovar. Revista de Ciencias Administrativas y Sociales, 24 (54), 75-88.

Grindle, M. (2007). Going Local: Decentralization, Democratization, and the Promise of Good Governance. Princeton: Princeton University Press.

Hevia, F. (2014). Peticiones, Protestas y Participación. Patrones de Relación Sociedad-Gobierno en la Educación Básica en Veracruz a Inicios del Siglo XXI. México: CIESAS.

- (2015). Construcción de Capacidades Estatales y Patrones de Relación Gobierno-Ciudadanos en México: un Análisis del Nivel Subnacional. Revista del CLAD. Reforma y Democracia, 62, 107-34.

Hevia, F. y Aguilera de Hombre, F. (2015). Gobierno Abierto en el Estado de Veracruz: Una Experiencia en Construcción. En Luna Pla, I., Bojórquez, J. y Hofmann, A. (Eds.), Gobierno Abierto: El Valor Social de la Información Pública. México: ITAIP, IIJ-UNAM.

Hofmann, A., Ramírez-Alujas, A. y Bojórquez, J. (Eds.). (2012). La Promesa del Gobierno Abierto. México: ITAIP, INFO-DF.

Instituto del Derecho a las Telecomunicaciones (IDET). (2015). Día del Internet: Promesas y Realidades. Disponible en http://www.idet.org. $\mathrm{mx} /$ comunicados/2015/dia-del-internet-promesas-y-realidades/ [0106-2017].

Instituto Nacional de Estadística y Geografía (INEGI). (2011). Principales Resultados del Censo de Población y Vivienda 2010. México: INEGI. 
(2016). Encuesta Nacional sobre Disponibilidad y Uso de Tecnologías de la Información en los Hogares 2015. Disponible en http://www.inegi.org.mx/est/contenidos/proyectos/encuestas/hogares/ regulares/dutih/2015/default_t.aspx [01-06-2017].

Kaufman, E. (2015). Lecciones Aprendidas de la Experiencia Internacional. Lo Bueno y lo Malo. En Luna Pla, I., Bojórquez, J. y Hofmann, A. (Eds.), Gobierno Abierto: El Valor Social de la Información Pública. México: ITAIP, IIJ-UNAM.

Lathrop, D. y Ruma, L. (Eds.). (2010). Open Government: Collaboration, Transparency, and Participation in Practice. Sebastopol: O'Reilly Media.

Luna Pla, I., Bojórquez, J. y Hofmann, A. (Eds.). (2015). Gobierno Abierto: El Valor Social de la Información Pública. México: ITAIP, IIJ-UNAM.

McDermott, P. (2010). Building Open Government. Government Information Quarterly, 27 (4), 401-413.

Mesina, J. (2013). Open Government Partnership en México y Brasil: La transparencia como Responsabilidad Compartida. México: INAP, GLAP, IICA.

Obama, B. (2009). Transparency and Open Government. Washington: The Press Office.

Open Government Partnership (OGP). (2016). Open Government Partnership. Disponible en http://www.opengovpartnership.org/ [0106-2017].

Olvera, A., Zavaleta, A. y Andrade, V. (Eds.), (2013). Violencia, inseguridad y justicia en Veracruz. Xalapa: Universidad Veracruzana.

Órgano de Fiscalización Superior del Estado de Veracruz (ORFIS). (2016). Gobierno Abierto. Disponible en http://www.orfis.gob.mx/ GobiernoAbierto/Galerias/03-02-2016/2015.html [01-06-2017].

Ostrom, E. (2015). Comprender la Diversidad Institucional. México: Fondo de Cultura Económica.

Oszlak, O. (2015). Gobierno Abierto: el Rumbo de los Conceptos. En Luna Pla, I., Bojórquez, J. y Hofmann, A. (Eds.), Gobierno Abierto: El Valor Social de la Información Pública. México: ITAIP, IIJ-UNAM. 
Parks, W. (1957). Open Government Principle: Applying the Right to Know under the Constitution. The George Washington Law Review, 26 (1), 1-77.

Prieto-Martín, P. y Ramírez-Alujas, A. (2014). Caracterizando la Participación Ciudadana en el Marco del Gobierno Abierto. Revista del CLAD. Reforma y Democracia, 58, 61-100.

Quintanilla, G. y Gil-Garcia, J. (2016). Gobierno Abierto y Datos Vinculados: Conceptos, Experiencias y Lecciones con Base en el Caso Mexicano. Revista del CLAD. Reforma y Democracia, 65, 69-102.

Sánchez, J. (2015). La Participación Ciudadana como Instrumento del Gobierno Abierto. Espacios Públicos 18 (43): 51-73.

Sandoval-Almazan, R. (2015). Gobierno Abierto y Transparencia: Construyendo un Marco Conceptual. Convergencia, 22 (68), 203-227.

Sandoval-Almazan, R. y Gil-Garcia, J. (2016). Toward an Integrative Assessment of Open Government: Proposing Conceptual Lenses and Practical Components. Journal of Organizational Computing and Electronic Commerce, 26 (1-2), 170-192.

Secretariado Técnico Local Veracruz. (2015a). Acta de Instalación del Secretariado Técnico del Estado de Veracruz de Ignacio de la Llave, para la implementación del Plan de Acción Local de Gobierno Abierto. Xalapa: Secretariado Técnico Local de Gobierno Abierto Veracruz.

- (2015b). Hacia el Plan de Acción Local. Disponible enhttp:/www.veracruzgobiernoabierto.com.mx/2015/04/17/acta-deinstalacion-del-secretariado-tecnico-local/. [01-06-2017].

Ugalde, Luis Carlos. (2015). La Democracia Interactiva Posible en México. En Luna Pla, I., Bojórquez, J. y Hofmann, A. (Eds.), Gobierno Abierto: El Valor Social de la Información Pública. México: ITAIP, IIJ-UNAM.

Valenzuela, R. (2013). El Federalismo Cooperativo como Factor Catalizador de un Gobierno Abierto. Revista Mexicana de Ciencias Politicas y Sociales 58 (219), 19-44.

Wang, H y Lo, J. (2016). Adoption of Open Government Data among Government Agencies. Government Information Quarterly, 33 (1), 8088. 
Wirtz, B. y Birkmeyer, S. (2015). Open Government: Origin, Development, and Conceptual Perspectives. International Journal of Public Administration, 38 (5), 381-396.

Yacuzzi, E. (2005). El Estudio de Caso como Metodología de Investigación: Teoría, Mecanismos Causales, Validación. Buenos Aires: Universidad del CEMA.

Yu, H. y Robinson, D. (2012). The New Ambiguity of 'Open Government. 59 UCLA Law Review Discourse, 178, 178-207

Recibido: 29-11-2016

Aceptación de la versión final: 12-06-2017 